\title{
miR-15a represses cancer cell migration and invasion under conditions of hypoxia by targeting and downregulating Bcl-2 expression in human osteosarcoma cells
}

\author{
JIALI LENG $^{1}$, QINGXU SONG ${ }^{2}$, YUGUANG ZHAO ${ }^{3}$ and ZHENYU WANG ${ }^{2}$ \\ ${ }^{1}$ Nursing Platform of Bone, Joint and Sports Medicine, ${ }^{2}$ Department of Spinal Surgery, \\ ${ }^{3}$ Cancer Center, The First Hospital of Jilin University, Changchun, Jilin 130021, P.R. China
}

Received October 19, 2017; Accepted December 12, 2017

DOI: $10.3892 /$ ijo.2018.4285

\begin{abstract}
Osteosarcoma is a common, high-risk primary bone malignancy that mostly affects the younger population. There has been no marked improvement in the clinical outcomes of osteosarcoma patients to date, and cancer recurrence and metastasis are common in high-grade osteosarcoma. Therefore, identifying new biomarkers and novel therapeutic targets is crucial for improving the prognosis of osteosarcoma patients. In the present study, the MG63 human osteosarcoma cell line was employed to examine the role of microRNA (miR)-15a in regulating cellular activities under hypoxic conditions. It was demonstrated that hypoxia stimulates migration and invasion in MG63 cells, which was correlated with the downregulation of miR-15a and upregulation of B-cell lymphoma 2 (Bcl-2) expression. Introduction of miR-15a or knockdown of endogenous $\mathrm{Bcl}-2$ may reduce hypoxia-induced cell invasion and migration through the regulation of matrix metalloproteinases. Analysis of the expression of miR-15a indicated that hypoxia repressed the transcription of deleted in lymphocytic leukemia 2 (DLEU2), which is the host gene of miR-15a. These findings indicated that miR-15a may be a valuable target for the treatment of osteosarcoma, particularly for patients with high-grade cancer or heavy tumor burden.
\end{abstract}

\section{Introduction}

Oxygen levels are crucial for cell survival. Hypoxia (insufficient oxygen) is always present in tumor areas due to the excessive proliferation of tumor cells and the increasing oxygen consumption. For solid tumors, hypoxia plays an

Correspondence to: Professor Zhenyu Wang, Department of Spinal Surgery, The First Hospital of Jilin University, 71 Xinmin Street, Changchun, Jilin 130021, P.R. China

E-mail: jluwangzy@163.com

Key words: microRNA-15a, osteosarcoma, cell migration, cell invasion, hypoxia important role in promoting tumor development and disease progression via favoring the formation of a neoplastic microenvironment.

Osteosarcoma is a common, high-risk primary bone malignancy that mostly presents in the first or second decades of life (1). Surgery and chemotherapy are the main treatment strategies for osteosarcoma. However, for high-grade osteosarcoma, the combination of surgery and chemotherapy does not always yield satisfactory outcomes due to the high rate of cancer recurrence and metastasis to the lungs $(2,3)$. There has been no marked improvement in the clinical outcomes of osteosarcoma patients to date. This stagnation in therapeutic advances may be attributed to the genetic, epigenetic and biological complexities of this tumor (4). Therefore, identifying new biomarkers and novel therapeutic targets is crucial for improving the prognosis of patients with osteosarcoma.

MicroRNAs (miRNAs/miRs) are a family of endogenous single-stranded, small non-protein-coding RNAs that affect fundamental cellular behaviors in diverse organisms. Mature miRNAs are $22 \mathrm{nt}$ long and regulate gene expression at the post-transcriptional level by inhibiting the translation and/or decreasing of the stability of target mRNAs $(5,6)$. Several hundred miRNAs have been identified and studied thus far, and they have been found to play key roles in various biological and pathological processes, such as cell proliferation, apoptosis, differentiation, metabolism, autophagy, migration and invasion (7-11).

miR-15a, located at $13 \mathrm{q} 14.3$, was identified as a tumor suppressor gene in chronic lymphocytic leukemia in 2002 (12). Later investigations indicated that miR-15a exerts its antitumor effects through targeting several oncogenes that are associated with cancer, such as CCND1 (encoding cyclin D1), WNT3A and B-cell lymphoma 2 (Bcl-2) $(13,14)$. To date, deletion and/or downregulation of miR-15a have been reported in several types of cancer, including breast, colorectal and lung cancer, as well as osteosarcoma $(11,15-17)$. Tian et al examined 45 pairs of human osteosarcoma samples and demonstrated that miR-15a expression was downregulated compared with that in corresponding adjacent normal tissues (11). However, the role of miR-15a in osteosarcoma tumor invasion and migration, particularly under hypoxic conditions, remains largely unknown. 
The aim of the present study was to investigate the role of miR-15a in regulating hypoxia-induced cell invasion and migration in human osteosarcoma cells, as well as the involvement of $\mathrm{Bcl}-2$ in this process and the underlying mechanism, in order to determine whether miR-15a may be of value as a therapeutic target for the treatment of osteosarcoma, particularly in patients with high-grade cancer or heavy tumor burden.

\section{Materials and methods}

Cell culture. The human osteosarcoma cell lines MG63 and U-2 OS, and the human osteoblast cell line hFOB1.19, were obtained from American Type Culture Collection (Manassas, VA, USA). MG63 and U-2 OS cells were maintained in Dulbecco's modified Eagle's medium (DMEM, Biological Industries, BI, Shanghai, China) supplemented with $10 \%$ fetal bovine serum (FBS; BI, Kibbutz Beit-Haemek, Israel) and streptomycin $(100 \mathrm{mg} / \mathrm{ml}) /$ penicillin $(100 \mathrm{U} / \mathrm{ml}$; HyClone, Beijing, China). U-2 OS cells were maintained in DMEM/Ham's F12 medium supplemented with 10\% FBS and streptomycin/penicillin. Cells were incubated at $37^{\circ} \mathrm{C}$ with $5 \% \mathrm{CO}_{2}$ and $20 \% \mathrm{O}_{2}$ in a humidified incubator (Thermo Fisher Scientific, Waltham, MA, USA).

Hypoxic culture. For the hypoxic culture, tissue culture plates were placed in a $37^{\circ} \mathrm{C}$ humidified $\mathrm{CO}_{2}(5 \%) / \mathrm{O}_{2}(1 \%) / \mathrm{N} 2(94 \%)$ incubator (Fisher Scientific Forma; Thermo Fisher Scientific).

$\alpha$-amanitin treatment of MG63 cells. MG63 cells were cultured to $70-80 \%$ confluence and treated with $100 \mu \mathrm{g} / \mathrm{ml} \alpha$-amanitin (Sigma-Aldrich; Merck KGaA, St. Louis, MO, USA) for $3 \mathrm{~h}$ to block nascent pri-miRNA synthesis. Subsequently, the cells were cultured under hypoxic or normoxic conditions for another 1, 2, 4 or $8 \mathrm{~h}$. Cells were collected and total RNA was extracted for the quantitative polymerase chain reaction (qPCR) assay.

miRNA, siRNAandcelltransfection.miR-15amimics(sequence: 5'-uagcagcaca uaaugguuugug-3') and the miRNA scrambles (sequence: 5'-UUGUACUAC ACAAAAGUACUG-3', control, CT) were purchased from GenePharma Co. Ltd. (Shanghai, China). For miRNA transfection, MG63 cells were transfected with Lipofectamine 2000 (Invitrogen; Thermo Fisher Scientific). According to the manufacturer's protocol, $100 \mathrm{pmol}$ miRNA mimics or miRNA scrambles were added to each well of a 6-well plate. For DNA transfection, $2.5 \mu \mathrm{g}$ plasmid DNA was transfected with Lipofectamine 2000 (Invitrogen; Thermo Fisher Scientific) in each well of a 6-well plate.

To knock down the level of Bcl-2, MG63 cells were transfected with 25 pmol Bcl-2 siRNA or non-targeting (NT) negative control siRNA (siRNA ID: 214532, Thermo Fisher Scientific) using Lipofectamine RNAiMAX transfection reagent (Thermo Fisher Scientific) following the manufacturer's instructions. Knockdown efficiency was measured by western blotting. All the cell experiments were performed at 24 post-transfection.

Cell invasion assay. For the cell invasion assay, 1x10 ${ }^{5}$ MG63 cells or the transfected cells were seeded in serum-free medium in a Transwell migration chamber $(8-\mu \mathrm{m}$ pore size; Corning, Shanghai, China). The upper chamber was coated with Matrigel (BD Biosciences, San Jose, CA, USA). DMEM containing $10 \%$ FBS was loaded to the lower well. After a 24-h culture in the hypoxic incubator, the cells that did not invade through the pores were carefully wiped off with a cotton swab. The cells located on the lower surface of the chamber were fixed and stained with $0.1 \%$ crystal violet (Sigma-Aldrich; Merck $\mathrm{KGaA}$ ) at room temperature and counted under a light microscope (Olympus, Tokyo, Japan) from five random fields. These experiments were repeated three times.

Migratory behavior assay. For the cell migration assay, MG63 cells or the transfected cells were seeded on a fibronectinprecoated CELLview cell culture dish (Greiner Bio-One, Stuttgart, Badenia-Wirtembergia, Germany) and cultured for $12 \mathrm{~h}$ in the hypoxic incubator. Cell images were captured at 10-min intervals with a CCD camera for $6 \mathrm{~h}$ to acquire image stacks. Each image stack was quantitatively analyzed by NIH ImageJ software.

RNA extraction, reverse transcription $(R T)$ and $q P C R$ analysis. Total RNA was extracted by Qiagen RNeasy Mini Kit (Qiagen, Shanghai, China) and reverse-transcribed to cDNA using Moloney murine leukemia virus reverse transcriptase (M-MLV, Invitrogen; Thermo Fisher Scientific). For Bcl-2 mRNA detection, $800 \mathrm{ng}$ total RNA was reverse-transcribed using oligo(dT) primers and random hexamer primers. For deleted in lymphocytic leukemia 2 (DLEU2) RNA detection, $800 \mathrm{ng}$ total RNA was reverse-transcribed using gene-specific reverse primer (GSRP). For microRNA detection, 1,500 ng total RNA was reverse-transcribed using microRNA-specific reverse primers (MSRP). The levels of $\beta$-actin and U6 were used to normalize gene and microRNA expression. The GSRP sequence was as follows: 5'-TCTCATACA GGTTACAGTTC-3'. The MSRP sequences were as follows: U6 5'-GTCGTATCCA GTGCAGGGTCCGAGGTATTCGCACTGGATACGACAA AATA-3'; miR-15a 5'-GTCGTATCCAGTGCAGGGTCCG AGGTATTCGCACTGGATACGACCACAAA-3'. qPCR analysis for the Bcl-2 and DLEU2 genes and miR-15a was performed using an aliquot of first-strand cDNA as the template in a $20-\mu 1$ reaction system containing $10 \mu 12 X$ SYBR premixed buffer (Roche, Shanghai, China), $2 \mu \mathrm{l}$ forward and reverse primers. The primers were as follows: $\mathrm{Bcl}-2$ forward, 5'-CTGCACCTGACGCCCTTCACC-3' and reverse, 5'-CAC ATGACCCCACCGAACTCAAAGA-3'; DLEU2 forward, 5'-TCTGGAGAACAGCCTCACTTC-3 and reverse, 5'-TGCT GAGCTAAGTAGAGGTCTC-3' (18); $\beta$-actin forward, 5'-CTG GCTCCTAGCACCATGAAGAT-3' and reverse, 5'-GGTGGA CAGTGAGGCCAGGAT-3' (19); U6 forward, 5'-CTCGCTTC GGCAGCACATA-3' and reverse, 5'-CAGTGCAGGGTCCG AGGTA-3'; miR-15a forward, 5'-CGCCTAGCAGCACATAA TGG-3' and reverse, 5'-AGTGCAGGGTCCGAGGTAT-3' (20). The PCR amplification process was as follows: 5 min denaturation at $95^{\circ} \mathrm{C}$ followed by 40 cycles at $95^{\circ} \mathrm{C}$ for $20 \mathrm{sec}, 58^{\circ} \mathrm{C}$ for $15 \mathrm{sec}$, and $72^{\circ} \mathrm{C}$ for $15 \mathrm{sec}$ (ABI StepOnePlus, Beijing, China). Data analysis was performed using the $2^{-\Delta \Delta \mathrm{Cq}}$ method.

Western blot analysis. Protein was extracted from MG63 cells with RIPA buffer (KeyGen Biotech. Co., Ltd., Nanjing, 
China) supplemented with cocktail protease inhibitor (Roche). Equal amounts of protein from each sample were separated by $12 \%$ sodium dodecyl sulfate polyacrylamide gel electrophoresis and transferred to polyvinylidene fluoride membranes (EMD Millipore, Billerica, MA, USA). The blots were then blocked with 5\% BSA (Sigma-Aldrich; Merck KGaA) in TBST at $37^{\circ} \mathrm{C}$ for $1 \mathrm{~h}$ and incubated with primary antibodies [Bcl-2 rabbit polyclonal antibody, 1:1,000, ab59348; hypoxiainducible factor (HIF)-1 $\alpha$ mouse monoclonal antibody, 1:1,000, ab1; Abcam, Shanghai, China. Matrix metalloproteinase (MMP)-2 rabbit monoclonal antibody, 1:1,000, 40994; MMP-9 rabbit monoclonal antibody, 1:1,000, 13667; Cell Signaling Technology, Shanghai, China] overnight at $4^{\circ} \mathrm{C}$. After washing 3 times in TBST, the membranes were incubated with horseradish peroxidase-coupled goat anti-rabbit or goat anti-mouse secondary antibody (sc-2004 and sc-2005, 1:3,000, Santa Cruz Biotechnology, Shanghai, China) at room temperature for $1 \mathrm{~h}$. The chemiluminescence signals were detected with a chemiluminescence system (ECL; EMD Millipore). Densitometric analysis was conducted using Quantity One software, version 4.6 (Bio-Rad Laboratories, Inc., Hercules, CA, USA).

Construction of luciferase reporter vectors. For luciferase reporter vector construction, a 3'UTR segment of $546 \mathrm{bp}$ of the 3'UTR of the Bcl-2 gene (UTR-WT) was amplified by PCR from human genomic DNA and inserted into the pGL3-control vector (Promega Corporation, Beijing, China), using the $\mathrm{XbaI}$ site downstream from the stop codon of luciferase. The following primers were used to generate pGL3-UTR-WT: UTR-WT forward, 5'-CTAGTCTAGAGCCTCAGGGAAC AGAATGATCAG-3' and reverse, 5'-CTAGTCTAGAAAGCG TCCACGTTCTTCATTG-3' (21). We also generated a luciferase reporter plasmid with mutant Bcl-2 3'UTR (UTR-MU) by using the Fast Site-Directed Mutagenesis kit (Tiangen, Beijing, China). The following primers were used to generate pGL3-UTR-MU: UTR-MU forward, 5'-ATCCAATCCTGA GGTCCGATCCTGCCAAA-3' and reverse, 5'-TTTGGCAGG ATCGGACCTCAGGATTGGAT-3'. Both UTR-WT and UTR-MU were confirmed by sequencing.

Luciferase activity assay. Briefly, 1×105 MG63 cells were co-transfected with $0.5 \mu \mathrm{g}$ pGL3-UTR-WT or pGL3UTR-MU luciferase vectors and 50 pmol miRNAs using Lipofectamine 2000 in 24-well plates. Renilla luciferase reporter plasmid pRL-SV40 (0.05 $\mu \mathrm{g}$, Promega Corporation) was co-transfected for monitoring transfection efficiency. Approximately $24 \mathrm{~h}$ later, cells were rinsed with phosphate-buffered saline and lysed with passive lysis buffer. Luminescence was measured by Dual-Luciferase Reporter assay system (Glomax 20/20, Promega Corporation). The luciferase activity was calculated as the ratio between the luminescence of firefly luciferase and the Renilla luciferase. Each experiment was repeated in triplicate.

Statistical analysis. Statistical analysis was conducted with SPSS 17.0 software (SPSS Inc., Chicago, IL, USA). All data were expressed as arithmetic mean \pm standard deviation. Statistical analysis was performed with one-way analysis of variance or Student's t-test. Results were considered statistically significant for P-values $<0.05$.

\section{Results}

Hypoxia represses miR-15a expression and stimulates MG63 cell invasion. In order to understand the expression of miR-15a in osteosarcoma cells, its levels in two osteosarcoma cell lines, MG63 and U-2 OS, were measured by qPCR. As a comparison, we also measured the level of miR-15a in the normal osteoblast cell line hFOB1.19. As shown in Fig. 1A, the level of miR-15a in the two osteosarcoma cell lines was significantly lower compared with that in the normal osteoblast cell line $(\mathrm{P}<0.05)$.

We further selected the MG63 cell line to investigate the effect of hypoxia on miR-15a expression and cell invasion in human osteosarcoma. MG63 cells were placed in a hypoxic incubator with $1 \% \mathrm{O}_{2}$ and cultured for $24 \mathrm{~h}$. To determine whether the hypoxia was effective, the expression of HIF-1 $\alpha$ was measured, and the western blotting results demonstrated that the level of HIF-1 $\alpha$ in MG63 cells was significantly increased (Fig. 1B). The miR-15a level under hypoxic stress was also measured by qPCR. Compared with the cells cultured under normoxic conditions, the level of miR-15a in MG63 cells cultured in hypoxia decreased significantly (Fig. 1A, P<0.05).

The invasion ability of MG63 cells was measured by the Transwell experiment and cells migrating through the Transwell chamber inserts were counted. Following exposure to hypoxia, the number of migrated cells was significantly increased compared with the normoxia group $(\mathrm{P}<0.05$, Fig. 1C). We also detected the expression of $\mathrm{Bcl}-2$ and matrix metalloproteinases by western blotting, and found that the levels of Bcl-2, MMP-2 and MMP-9 were significantly increased (Fig. 1D and E) under hypoxic conditions.

Overexpression of miR-15a represses the invasion and migration of MG63 cells under hypoxia. To investigate whether the hypoxia-induced cell invasion is correlated with the downregulation of miR-15a, we introduced exogenous miR-15a into MG63 cells by transfection of miR-15a mimics. At $48 \mathrm{~h}$ post-transfection, the total RNA was extracted and the level of miR-15a was measured by qPCR. As shown in Fig. 2A, the total miRA-15a was markedly increased after the transfection $(\mathrm{P}<0.05)$. Subsequently, the invasion ability of the transfected MG63 cells under hypoxic condition was measured. After the transfected MG63 cells were exposed to hypoxia for $24 \mathrm{~h}$, the number of cells migrating through the Transwell chamber inserts was counted and illustrated. As shown in Fig. 2B, the number of migrated cells under hypoxia was markedly decreased after miR-15a was overexpressed $(\mathrm{P}<0.05)$. We also analyzed the migratory behavior of the transfected MG63 cells by tracking the migration paths and analyzing the accumulated distance of the individual cells. As shown in Fig. 2C, the average accumulated distance of the miR-15a-transfected cells was $26.1 \mu \mathrm{m}$, whereas the average accumulated distance of the miR-scramble-transfected cells was $51.2 \mu \mathrm{m}(\mathrm{P}<0.05)$. The cancer cell invasion and migration required MMP-2 and MMP-9, which were also detected by western blotting, and the results demonstrated that introduction of miR-15a significantly suppressed the expression of MMP-2 and MMP-9 under hypoxic conditions (Fig. 2D, $\mathrm{P}<0.05$ ).

Knockdown of Bcl-2 represses cell invasion and migration in MG63 cells under hypoxia. Since hypoxia can stimulate 
A

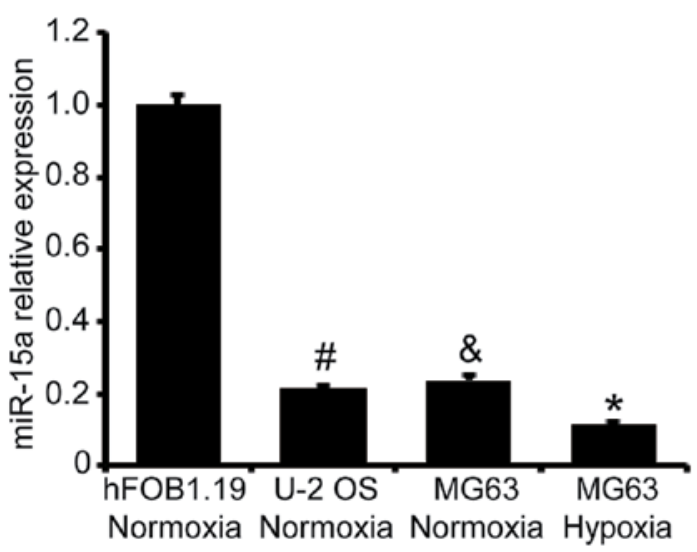

B

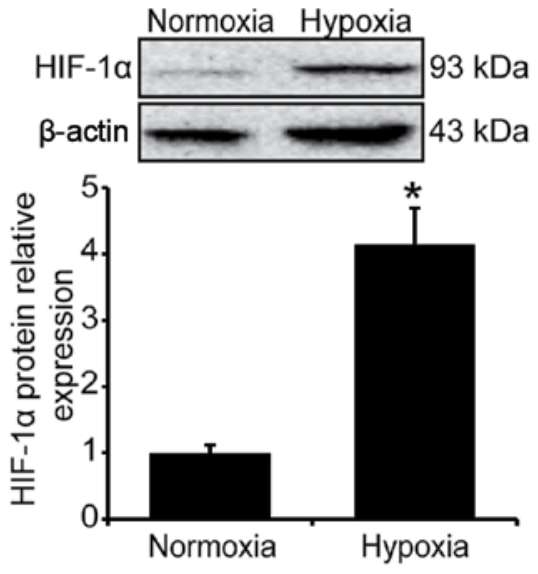

C
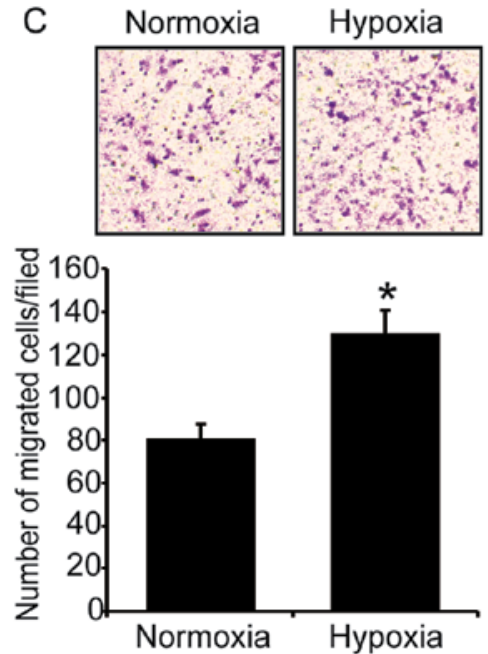

D
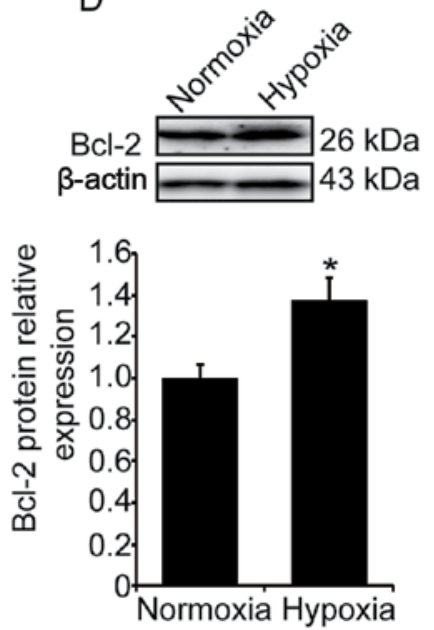

E
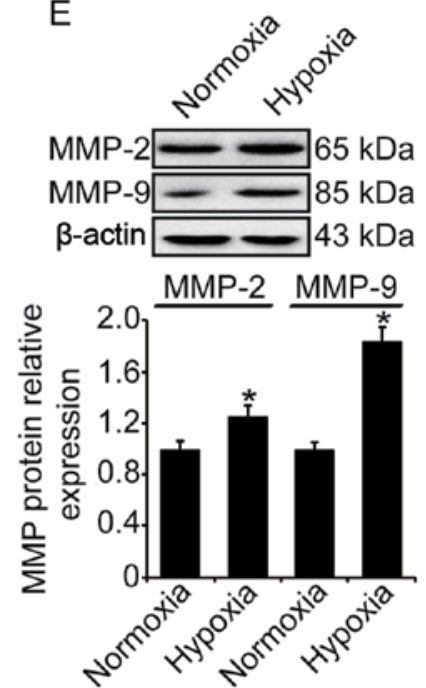

Figure 1. Hypoxia represses miR-15a expression and stimulates cell invasion in osteosarcoma. The levels of miR-15a in two human osteosarcoma cell lines (MG63 and U-2 OS) and one human osteoblast cell line (hFOB1.19) were measured and compared. MG63 cells were exposed to hypoxia (1\% ( $_{2}$ ) for 24 h. The expression of miR-15a was measured by qPCR, the expression of HIF-1 $\alpha$, Bcl-2 and MMPs was measured by western blotting, and the cell invasion ability was measured by the Transwell assay. (A) Compared with the normal osteoblast cells, the levels of miR-15a in osteosarcoma cells were significantly lower. Additionally, the level of miR-15a decreased significantly after MG63 cells were exposed to hypoxia. All the results were normalized to U6 and expressed as fold-change. Statistical results were based on one-way analysis of variance; ${ }^{\# \&} \mathrm{P}<0.05$ vs. normal osteoblast cells; $\mathrm{P}<0.05$ vs. MG63 cells cultured under normoxic conditions. (B) Hypoxia-induced HIF-1 $\alpha$ upregulation in MG63 cells. Upper panel, western blotting; lower panel, relative band density of western blotting. (C) The number of MG63 cells migrating through the Transwell chamber inserts was significantly increased after cells were exposed to hypoxia. Upper panel, invading MG63 cells were stained with crystal violet; lower panel, mean number of invading MG63 cells. (D) Hypoxia-induced Bcl-2 upregulation in MG63 cells. Upper panel, western blotting; lower panel, relative band density of the western blotting. (E) Hypoxia-induced upregulation of MMP-2 and MMP-9 in MG63 cells. Upper panel, western blotting; lower panel, relative band density of the western blotting. qPCR, quantitative polymerase chain reaction analysis; HIF-1 $\alpha$, hypoxia-inducible factor-1 $\alpha$; Bcl-2, B-cell lymphoma 2; MMP, matrix metalloproteinase.

the expression of endogenous Bcl-2, we used Bcl-2 siRNA to knock down the level of Bcl-2 and determine whether it will reduce the hypoxia-induced cell invasion and migration. As shown in Fig. 3A, following transfection of Bcl-2 siRNA, the level of Bcl-2 was significantly decreased $(\mathrm{P}<0.05)$. Additionally, compared with the control group, Bcl-2 knockdown significantly suppressed hypoxia-induced cell invasion and migration (Fig. 3B and C).

miR-15a represses the expression of $\mathrm{Bcl}-2$ at the posttranscriptional level. The miR-15a-transfected MG63 cells were exposed to hypoxia for $24 \mathrm{~h}$ and the expression of $\mathrm{Bcl}-2$ was examined at the transcriptional and post-transcriptional levels. The Bcl-2 mRNA expression was measured by qPCR, and there was no significant difference between the miR-15a and the miR-scramble transfection groups (Fig. 4A, P>0.05). However, the Bcl-2 protein level in the miR-15a transfection group was significantly decreased (Fig. 4B, P<0.05). These findings suggest that miR-15a may suppress the expression of Bcl-2 at the post-transcriptional level.

Bcl-2 mRNA is a direct target of miR-15a. It was reported that the Bcl-2 mRNA is a target of the miR-15 family (21), and our current experiment results also revealed that miR-15a suppresses the expression of $\mathrm{Bcl}-2$ at the post-transcriptional level. In order to confirm the direct binding of miR-15a to the Bcl-2 mRNA in MG63 cells, the 3'UTR fragment containing wild-type or mutant seed region of the miR-15a was cloned 
A

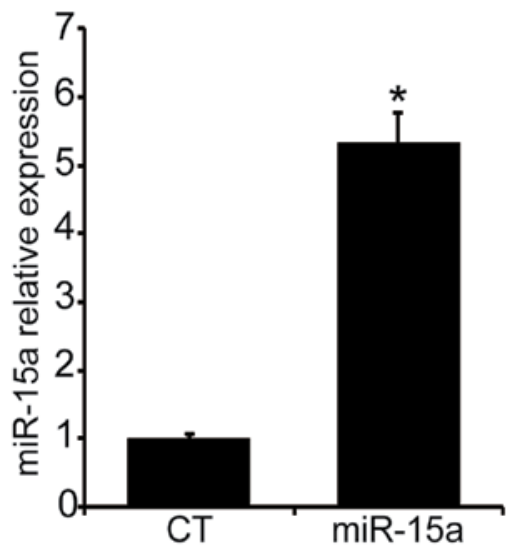

C
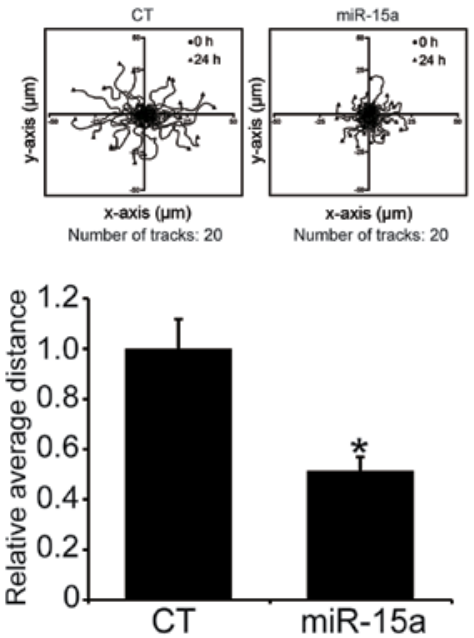

B
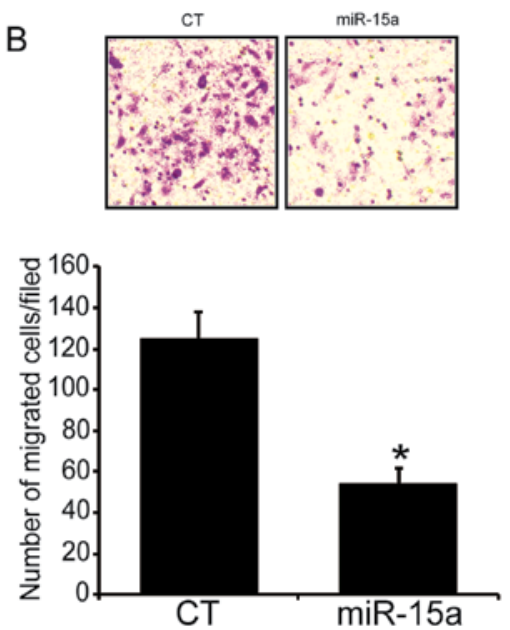

$\mathrm{D}$
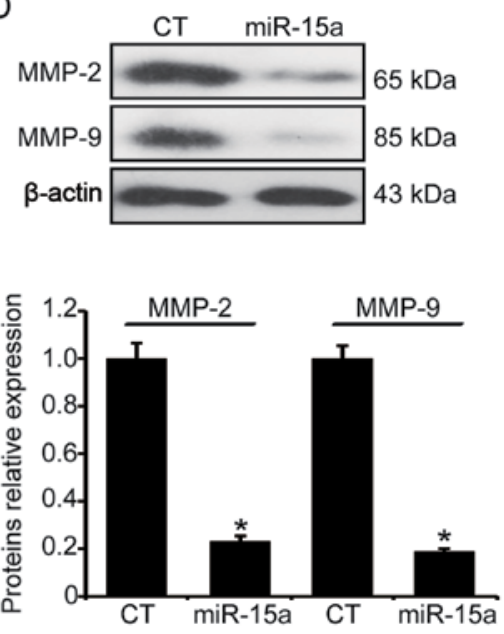

Figure 2. Overexpression of miR-15a inhibits the invasion and migration of MG63 cells under hypoxic conditions. Exogenous miR-15a was introduced into MG63 cells by transfection of miR-15a mimics using Lipofectamine 2000. After the transfection, the invasion and migration ability of the transfected MG63 cells under hypoxia was assessed by the Transwell assay and the tracking of the migration paths. (A) The level of total miR-15a was measured by qPCR. The results were normalized to U6 and expressed as fold-change relative to the control. (B) The number of cells migrating through the Transwell chamber inserts was markedly decreased. Upper panel, invading MG63 cells were stained by crystal violet; lower panel, mean number of invading MG63 cells. (C) Mean accumulated distance of the miR-15a-transfected MG63 cells. Upper panel, the cell migration paths were tracked by a CCD camera; lower panel, the accumulated distance of the individual cells was analyzed by ImageJ software. (D) MMP-2 and MMP-9 levels were determined by western blotting. Upper panel, western blotting; lower panel, relative band density of the western blotting. ${ }^{*} \mathrm{P}<0.05$ vs. the miR-scramble-transfection group. qPCR, quantitative polymerase chain reaction analysis; MMP, matrix metalloproteinase; CT, control.
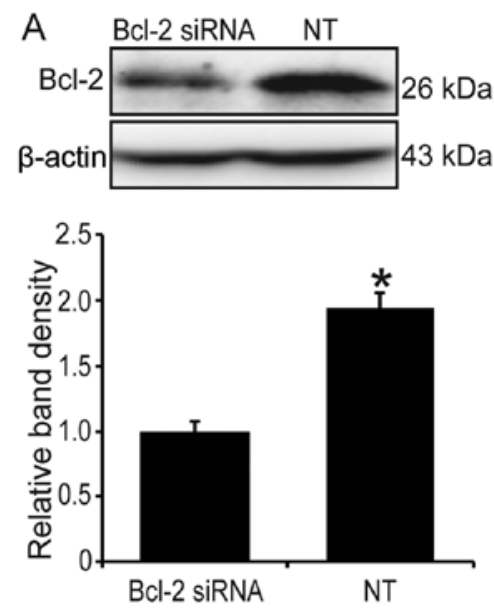

B

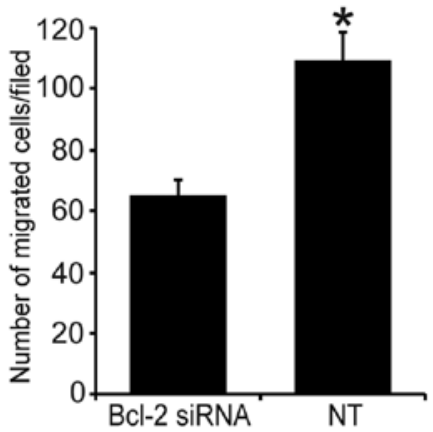

C

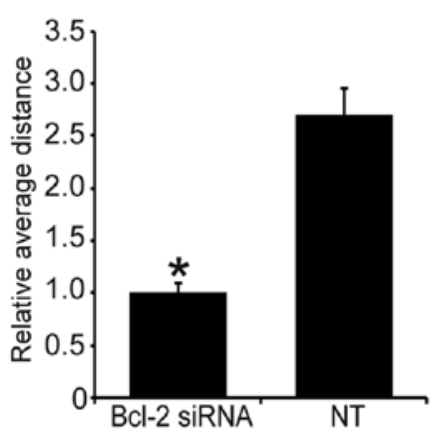

Figure 3. Knockdown of endogenous Bcl-2 inhibits the invasion of MG63 cells under hypoxic conditions. The level of Bcl-2 was knocked down by siRNA and the cell invasion and migration under hypoxia were assessed. (A) The western blotting results revealed that the expression level of Bcl-2 was significantly decreased. Upper panel, western blotting; lower panel, relative band density of the western blotting. (B) Knockdown of Bcl-2 suppressed the invasion of MG63 cells. (C) Knockdown of Bcl-2 suppressed the migration of MG63 cells. * $\mathrm{P}<0.05$ vs. the NT group. Bcl-2, B-cell lymphoma 2; NT, non-targeting. 
A

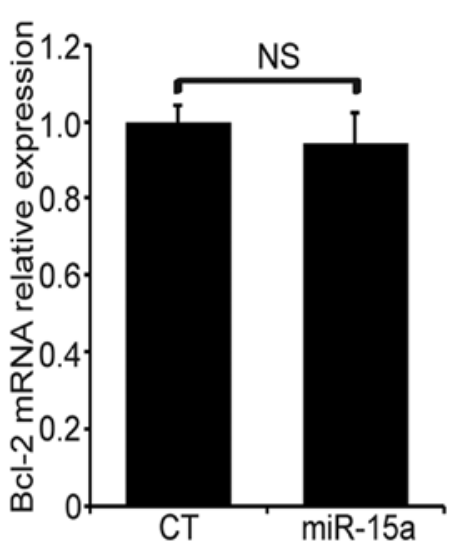

B
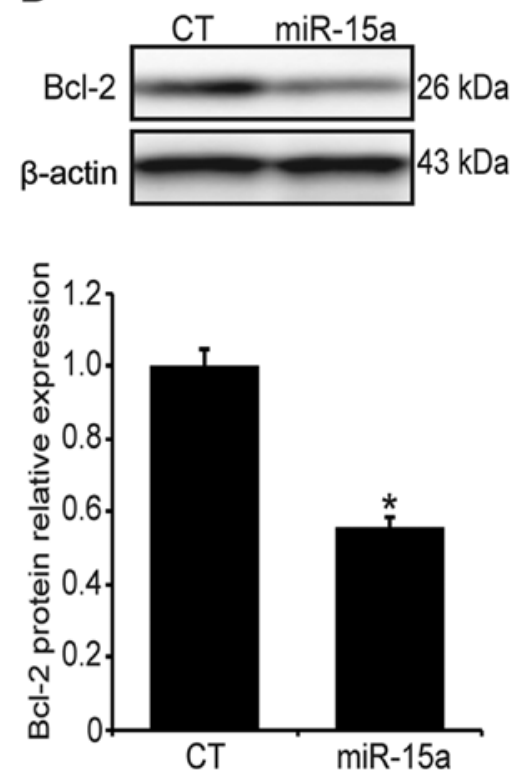

Figure 4. miR-15a downregulates Bcl-2 at the post-transcriptional level. The transfected MG63 cells were exposed to hypoxia for $24 \mathrm{~h}$. The Bcl-2 mRNA expression was measured by qPCR and the Bcl-2 protein level was measured by western blotting. (A) miR-15a transfection did not affect the Bcl-2 mRNA expression. The results were normalized to $\beta$-actin and expressed as fold-change relative to the control. (B) miR-15a transfection suppressed the Bcl-2 protein expression. Upper panel, western blotting; lower panel, relative band density of the western blotting. ${ }^{*} \mathrm{P}<0.05$ vs. miR-scramble-transfection group; NS, no significance compared with the miR-scramble-transfection group. Bcl-2, B-cell lymphoma 2; qPCR, quantitative polymerase chain reaction analysis CT, control.

A

$$
\begin{aligned}
& \text { Hsa_Bcl-2 3'UTR } \\
& \text { Hsa_miR-15a } \\
& \text { Hsa_Bcl-2 3'UTR-mu }
\end{aligned}
$$

\section{0-TATGGAATATCCAATCCTGTGCTGCTATCC-3290 \\ GTGTTTGGTAATACACGACGAT-5'}

3260-TATGGAATATCCAATCCTGAGGTCCGATCC-3290

B

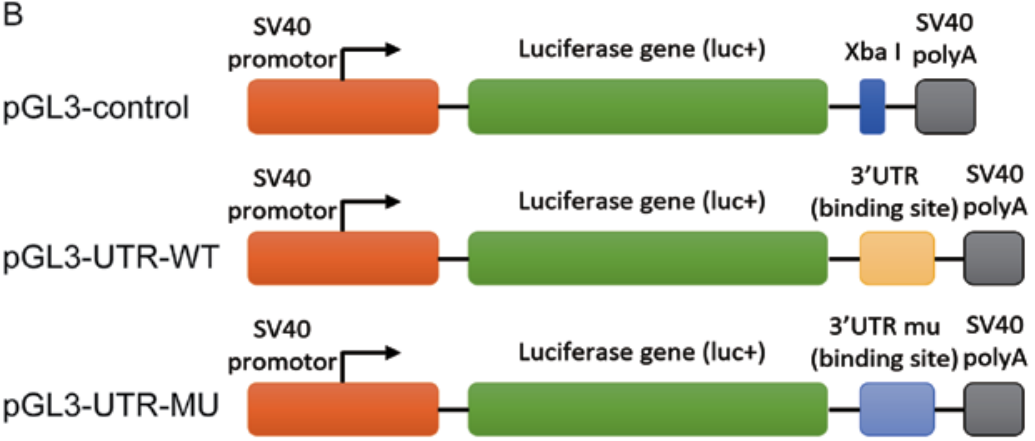

C

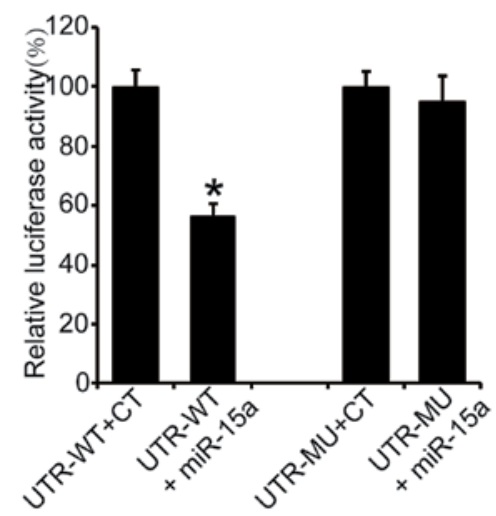

Figure 5. Bcl-2 mRNA is a direct target of miR-15a. The 3'UTR fragment containing wild-type or mutant seed region of the miR-15a was cloned into a dual-luciferase reporter vector and the luciferase activity was analysed. (A) Cloning of the wild-type and mutant 3'UTR of the Bcl-2 gene. (B) Construction of the luciferase reporter plasmids. (C) The wild-type reporter vector exhibited a significant reduction of luciferase activity when co-transfected with miR-15a, whereas the mutation vector abrogated this inhibitory effect. "P<0.05 vs. the UTR-WT + CT group. Bcl-2, B-cell lymphoma 2; UTR, untranslated region; WT, wild-type; MU, mutant; CT, control. 
A

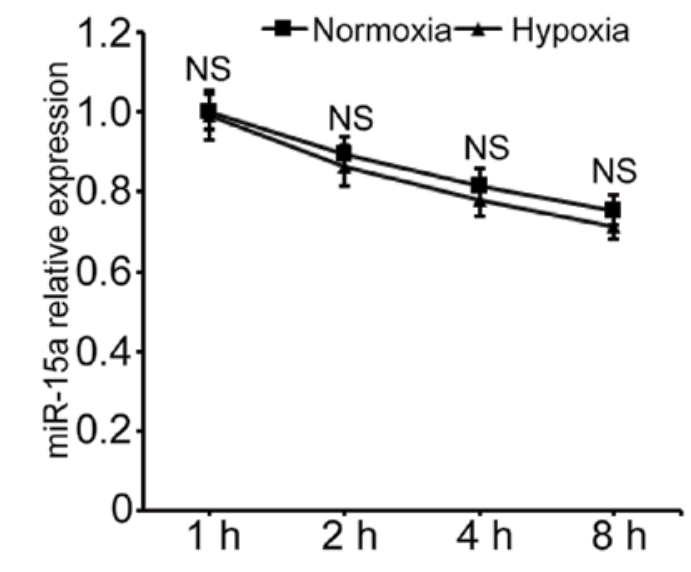

C

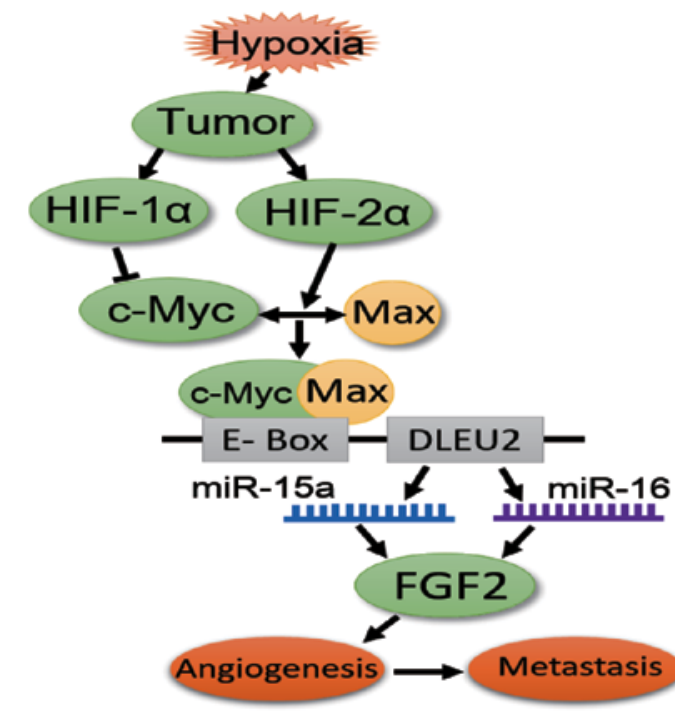

B
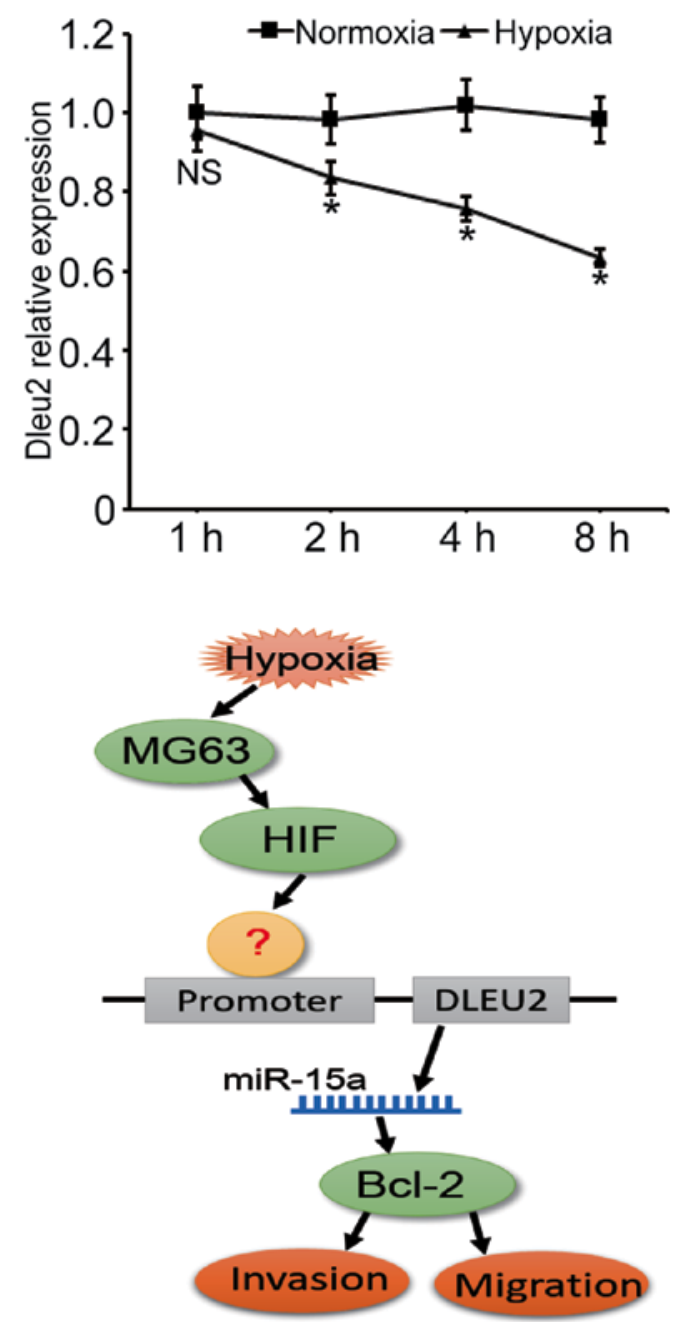

Figure 6. Hypoxia decreases the level of miR-15a through inhibiting the transcription of the DLEU2 gene. miR-15a expression under hypoxic stress was investigated at the transcriptional and post-transcriptional levels. (A) The degradation velocity of miR-15a was almost the same under hypoxic and normoxic conditions. MG63 cells were treated with $100 \mu \mathrm{g} / \mathrm{ml} \alpha$-amanitin for $3 \mathrm{~h}$ to block nascent pri-miRNA synthesis and cells were cultured under hypoxia or normoxia for another 1, 2, 4 or $8 \mathrm{~h}$. The miR-15a level was measured by qPCR. (B) The DLEU2 gene transcription level was significantly decreased under hypoxic stress. MG63 cells were cultured in hypoxia or normoxia, and the DLEU2 mRNA level was measured by qPCR at 1, 2, 4 or 8 h time points. (C) Sketch maps for the mechanisms of miR-15a-mediated cell activities under hypoxia. Left panel, c-Myc-HIF-mediated repression of miR-15-16 in hypoxia promotes tumor angiogenesis and metastasis (34); right panel, putative mechanism of miR-15a mediated cell invasion and migration in MG63 cells. DLEU2, deleted in lymphocytic leukemia 2; qPCR, quantitative polymerase chain reaction analysis; HIF, hypoxia-induciblefactor; FGF, fibroblast growth factor.

into a luciferase reporter vector (Fig. 5A and B). The luciferase activity assay revealed that the wild-type reporter vector exhibited a significant reduction of luciferase activity when co-transfected with miR-15a compared with the control group; by contrast, the mutation vector abrogated this inhibitory effect of miR-15a (Fig. 5C, $\mathrm{P}<0.05$ ). This result suggests that miR-15a suppresses Bcl-2 expression through direct binding to its 3 'UTR.

Hypoxia decreases DLEU2 transcription. After proving that hypoxia represses miR-15a expression in MG63 cells, we further investigated whether miR-15a was downregulated at the transcriptional or post-transcriptional level. MG63 cells were first treated with $\alpha$-amanitin to block the nascent primiRNA synthesis, and then the levels of miR-15a at 1, 2, 4 or $8 \mathrm{~h}$ under hypoxia were measured. As shown in Fig. 6A, following treatment of the MG63 cells with $\alpha$-amanitin, the level of miR-15a decreased by $\sim 25 \%$ after the cells were cultured for $8 \mathrm{~h}$, regardless of whether the cells were under hypoxic or normoxic conditions $(\mathrm{P}>0.05)$.

Since DLEU2 is the host gene of miR-15a, the mRNA levels of this gene under hypoxia were measured to estimate the transcriptional level of miR-15a. The qPCR results demonstrated that, after the cells were cultured in hypoxia for 2, 4 and $8 \mathrm{~h}$, the mRNA level of DLEU2 gene decreased by 15.0 , 25.6 and $35.6 \%$, respectively, compared with the normoxia control group. These findings suggest that hypoxia represses the level of miR-15a mainly at the transcriptional level.

\section{Discussion}

Inadequate oxygen supply is common in tissues harboring a heavy tumor burden. Tumor cells alter DNA transcription in response to hypoxic stress and, therefore, cell behaviors 
change. HIF family members are considered to be oxygen sensors, and HIF-involved hypoxia signaling pathways are among the most popular research targets. In addition to HIF, other genes, such as vascular endothelial growth factor, angiogenin, p53, nuclear factor- $\mathrm{kB}, \mathrm{p} 38$ and $\mathrm{Bcl}-2$, also play key roles in hypoxia-induced cellular activities (22-27).

Hypoxia promotes cell invasion, survival, angiogenesis and other cellular activities by modifying or regulating cellular molecules. miRNAs are recognized as a class of regulators affecting gene expression and are involved in several normal and pathological cellular processes. Numerous studies have demonstrated that miRNAs are correlated with various types of human cancer. In vivo studies demonstrated that restoration of miR-15a in tumors may ameliorate disease manifestations $(28,29)$. However, how miRNAs interact with certain conditions in the tumor microenvironment, such as hypoxia, has not yet been fully elucidated (30). In recent years, it was demonstrated that miRNAs play important roles in several pivotal signaling pathways that respond to hypoxia and are associated with hypoxic adaptation. For example, Zuo et al reported that miR-210 inhibits cell proliferation via inducing cell cycle arrest and apoptosis in human laryngeal carcinoma (31), whereas Li et al observed that miR-137 inhibits mitophagy via the regulation of the mitophagy receptors FUNDC1 and NIX (32).

Growing evidence indicates that the miR-15 family members, which include miR-15a, miR-15b, miR-16, miR-195, miR-424 and miR-497, are implicated in oncogenesis, cardiovascular and neurodegenerative diseases. Recent studies reported that miR-15a was also involved in hypoxia-induced gene expression regulation and cellular hypoxic adaptation. Yang et al found that hypoxia increases the level of miR-15a in rat cardiomyoblasts, and the inhibition of miR-15a significantly reduces cardiomyocyte apoptosis and the release of lactate dehydrogenase and malondialdehyde (33); Xue et al found that miR-15-16 transcription is repressed by hypoxia, which promotes tumor angiogenesis and hematogenous metastasis in tumor cells (34). We herein demonstrated that hypoxia enhances cell invasion and migration in the human osteosarcoma cell line MG63 through the downregulation of miR-15a and the upregulation of Bcl-2 (Fig. 1). However, as tumor cells are always heterogeneous and different types of tumors have distinct genetical backgrounds, more osteosarcoma cell lines and clinical osteosarcoma tissues should be investigated in future studies to draw more definitive conclusions.

$\mathrm{Bcl}-2$ is one of the key participants in the protection of cells from apoptotic death. Abnormally high levels of Bcl-2 occur in over half of all human cancers (35). In addition to conferring a survival advantage on cells, Bcl-2 is key to a wide variety of biological processes, including tumor invasion and metastasis $(36,37)$. For example, Ricca et al proved that Bcl-2 overexpression induces MMP-9 transcription and enhances cell metastatic potential in human MCF7 breast cancer cells (38). miRNAs have been estimated to regulate up to $30 \%$ of all human genes (39). However, how miRNAs regulate Bcl-2 during the process of tumor metastasis, particularly under hypoxic conditions, remains unclear. Wang et al reported that miR-429 inhibits cell invasion and promotes apoptosis by targeting Bcl-2 and SP1 in esophageal carcinoma cells (40); Wu et al reported that miR-16 inhibits cell proliferation, invasion and metastasis of HepG2 cells through upregulation of Bax, downregulation of Bcl-2, and decrease of MMP-2 and MMP-9 (41). In the present study, we demonstrated that miR-15a suppresses the expression of Bcl-2 at the post-transcriptional level and partially abolishes the hypoxiainduced cell invasion and migration (Figs. 2 and 4). Since the miR-15a and Bcl-2 mRNA sequences share a complementary homology, we demonstrated that Bcl-2 is a post-transcriptional target of miR-15a tumor suppressor, and miR-15a directly binds to the 3'UTR of Bcl-2 mRNA under hypoxia (Fig. 5). These results suggest that the interaction between miR-15a and Bcl-2 plays a pivotal role in hypoxia-induced MG63 cell invasion and migration. Our findings are consistent with those of Wang et al and Wu et al.

miR-15a has been recognized as one of the most important miRNAs in multiple human tissues, and has been identified as downregulated or deleted in several types of cancer; however, little is known on its transcriptional regulation. Since DLEU2 is the host gene of miR-15a, the level of mature miR-15a is associated with the transcription of DLEU2. Lerner et al reported that DLEU2 overexpression blocks cellular proliferation and inhibits the colony-forming ability of tumor cells in a miR-15a/miR-16-1-dependent manner (42); Xue et al reported that hypoxia promotes the heterodimerization of c-Myc/Max and downregulates the transcription of DLEU2/miR-15-16, which enhances angiogenesis and tumor metastasis (Fig. 6, left panel) (34). In the present study, we observed a similar phenomenon, in that hypoxia decreases the transcription of DLEU2/miR-15a. However, in our study, the target gene of miR-15a, which regulates cell invasion and migration, was Bcl-2 rather than FGF2 (Fig. 6, right panel). Moreover, the mechanism through which hypoxia stimulates the transcription of DLEU2 and whether HIF is involved in this process must be fully elucidated through further investigation.

In conclusion, our study demonstrated that low concentration of oxygen represses the transcription of the DLEU2 gene, decreases the level of miR-15a, and promotes MG63 cell invasion and migration. Overexpression of miR-15a represses cell migration and invasion via the regulation of Bcl-2 and MMPs. Our findings indicate that miR-15a may be a valuable target for the treatment of osteosarcoma, particularly in patients with high-grade cancer or heavy tumor burden. Of note, the present study is a preliminary study, and these results are based on an in vitro cell model. In order to obtain more solid experimental data, an in vivo investigation using an osteosarcoma-bearing nude mice model is required.

\section{Acknowledgements}

Not applicable.

\section{Funding}

This study was supported in part by grants from the National Science Foundation of China (no. 81670221 to Yuguang Zhao).

\section{Availability of data and materials}

The analysed data sets generated during the study are available from the corresponding authors on reasonable request. 


\section{Authors' contributions}

ZW contributed to the design of the study and wrote the manuscript. JL performed the experiments. QS analyzed the data. YZ helped perform the analysis with constructive discussions. All authors have read and approved this manuscript.

\section{Ethics approval and consent to participate}

Not applicable.

\section{Consent for publication}

Not applicable.

\section{Competing interests}

The authors declare that they have no competing interests.

\section{References}

1. Berner K, Johannesen TB and Bruland OS: Clinical epidemiology of low-grade and dedifferentiated osteosarcoma in Norway during 1975 and 2009. Sarcoma 2015: 917679, 2015.

2. Longhi A, Errani C, De Paolis M, Mercuri M and Bacci G: Primary bone osteosarcoma in the pediatric age: State of the art. Cancer Treat Rev 32: 423-436, 2006.

3. Anderson ME: Update on survival in osteosarcoma. Orthop Clin North Am 47: 283-292, 2016

4. Morrow JJ and Khanna C: Osteosarcoma genetics and epigenetics: Emerging biology and candidate therapies. Crit Rev Oncog 20: 173-197, 2015.

5. Van Wynsberghe PM, Chan SP, Slack FJ and Pasquinelli AE: Analysis of microRNA expression and function. Methods Cell Biol 106: 219-252, 2011.

6. Farh KK, Grimson A, Jan C, Lewis BP, Johnston WK, Lim LP, Burge CB and Bartel DP: The widespread impact of mammalian MicroRNAs on mRNA repression and evolution. Science 310: 1817-1821, 2005.

7. Mendell JT: MicroRNAs: Critical regulators of development, cellular physiology and malignancy. Cell Cycle 4: 1179-1184, 2005.

8. PourrajabF,BabaeiZarchM,BaghiYazdiM,HekmatimoghaddamS and Zare-Khormizi MR: MicroRNA-based system in stem cell reprogramming; differentiation/dedifferentiation. Int J Biochem Cell Biol 55: 318-328, 2014

9. Dumortier O, Hinault C and Van Obberghen E: MicroRNAs and metabolism crosstalk in energy homeostasis. Cell Metab 18 312-324, 2013.

10. Frankel LB and Lund AH: MicroRNA regulation of autophagy. Carcinogenesis 33: 2018-2025, 2012.

11. Tian X, Zhang J, Yan L, Dong JM and Guo Q: miRNA-15a inhibits proliferation, migration and invasion by targeting TNFAIP1 in human osteosarcoma cells. Int J Clin Exp Pathol 8: 6442-6449, 2015.

12. Calin GA, Dumitru CD, Shimizu M, Bichi R, Zupo S, Noch E, Aldler H, Rattan S, Keating M, Rai K, et al: Frequent deletions and down-regulation of micro- RNA genes miR15 and miR16 at $13 q 14$ in chronic lymphocytic leukemia. Proc Natl Acad Sci USA 99: 15524-15529, 2002.

13. Bonci D, Coppola V, Musumeci M, Addario A, Giuffrida R, Memeo L, D'Urso L, Pagliuca A, Biffoni M, Labbaye C, et al: The miR-15a-miR-16-1 cluster controls prostate cancer by targeting multiple oncogenic activities. Nat Med 14: 1271-1277, 2008.

14. Aqeilan RI, Calin GA and Croce CM: miR-15a and miR-16-1 in cancer: Discovery, function and future perspectives. Cell Death Differ 17: 215-220, 2010

15. Luo Q, Li X, Li J, Kong X, Zhang J, Chen L, Huang Y and Fang L: miR-15a is underexpressed and inhibits the cell cycle by targeting CCNE1 in breast cancer. Int J Oncol 43: 1212-1218, 2013.
16. Shi L, Jackstadt R, Siemens H, Li H, KirchnerT and Hermeking H: p53-induced miR-15a/16-1 and AP4 form a double-negative feedback loop to regulate epithelial-mesenchymal transition and metastasis in colorectal cancer. Cancer Res 74: 532-542, 2014.

17. Bandi N and Vassella E: miR-34a and miR-15a/16 are co-regulated in non-small cell lung cancer and control cell cycle progression in a synergistic and $\mathrm{Rb}$-dependent manner. Mol Cancer 10: 55, 2011.

18. Zhang H, Li L, Liu F, Zhang WY, Wei YZ, Wu XJ, Xi YY, Zhou YR, Chen HX and Lin YL: Proliferation, migration and invasion of cervical cancer cells. Xiandai Shengwu Yixue Jinzhan 16: 2801-2805, 2016 (In Chinese)

19. Liu G, Li Y and Gao XG: microRNA-181a is upregulated in human atherosclerosis plaques and involves in the oxidative stress-induced endothelial cell dysfunction through direct targeting Bcl-2. Eur Rev Med Pharmacol Sci 20: 3092-3100, 2016.

20. Zhu DX, Miao KR, Zhu YD, Zhu HY, Fan L, Liu P, Xu W and Li JY: Detection of miRNA levels in leukemia patients by real-time quantitative PCR. Zhongguo Shi Yan Xue Ye Xue Za Zhi 18: 757-761, 2010 (In Chinese).

21. Cimmino A, Calin GA, Fabbri M, Iorio MV, Ferracin M, Shimizu M, Wojcik SE, Aqeilan RI, Zupo S, Dono M, et al: miR-15 and miR-16 induce apoptosis by targeting BCL2. Proc Natl Acad Sci USA 102: 13944-13949, 2005.

22. Wu MZ, Chen SF, Nieh S, Benner C, Ger LP, Jan CI, Ma L, Chen $\mathrm{CH}$, Hishida T, Chang HT, et al: Hypoxia drives breast tumor malignancy through a TET-TNF $\alpha$-p38-MAPK signaling axis. Cancer Res 75: 3912-3924, 2015.

23. Deacon K, Onion D, Kumari R, Watson SA and Knox AJ: Elevated SP-1 transcription factor expression and activity drives basal and hypoxia-induced vascular endothelial growth factor (VEGF) expression in non-small cell lung cancer. J Biol Chem 287: 39967-39981, 2012

24. Hartmann A, Kunz M, Köstlin S, Gillitzer R, Toksoy A, Bröcker EB and Klein CE: Hypoxia-induced up-regulation of angiogenin in human malignant melanoma. Cancer Res 59: 1578-1583, 1999.

25. Timani KA, Liu Y, Fan Y, Mohammad KS and He JJ: Tip110 regulates the cross talk between $\mathrm{p} 53$ and hypoxia-inducible factor $1 \alpha$ under hypoxia and promotes survival of cancer cells. Mol Cell Biol 35: 2254-2264, 2015.

26. Liu L, Salnikov AV, Bauer N, Aleksandrowicz E, Labsch S, Nwaeburu C, Mattern J, Gladkich J, Schemmer P, Werner J, et al: Triptolide reverses hypoxia-induced epithelial-mesenchymal transition and stem-like features in pancreatic cancer by NF- $\mathrm{BB}$ downregulation. Int J Cancer 134: 2489-2503, 2014.

27. Shimizu S, Eguchi Y, Kosaka H, Kamiike W, Matsuda $H$ and Tsujimoto Y: Prevention of hypoxia-induced cell death by Bcl-2 and Bcl-xL. Nature 374: 811-813, 1995.

28. Kasar S, Salerno E, Yuan Y, Underbayev C, Vollenweider D, Laurindo MF, Fernandes H, Bonci D, Addario A, Mazzella F, et al: Systemic in vivo lentiviral delivery of miR-15a/16 reduces malignancy in the NZB de novo mouse model of chronic lymphocytic leukemia. Genes Immun 13: 109-119, 2012.

29. Dai L, Wang W, Zhang S, Jiang Q, Wang R, Dai L, Cheng L, Yang Y, Wei YQ and Deng HX: Vector-based miR-15a/16-1 plasmid inhibits colon cancer growth in vivo. Cell Biol Int 36: 765-770, 2012.

30. Shen G, Li X, Jia YF, Piazza GA and Xi Y: Hypoxia-regulated microRNAs in human cancer. Acta Pharmacol Sin 34: 336-341, 2013.

31. Zuo J, Wen M, Lei M, Peng X, Yang X and Liu Z: miR-210 links hypoxia with cell proliferation regulation in human Laryngocarcinoma cancer. J Cell Biochem 116: 1039-1049, 2015.

32. Li W, Zhang X, Zhuang H, Chen HG, Chen Y, Tian W, Wu W, Li Y, Wang S, Zhang L, et al: MicroRNA-137 is a novel hypoxiaresponsive microRNA that inhibits mitophagy via regulation of two mitophagy receptors FUNDC1 and NIX. J Biol Chem 289: 10691-10701, 2014.

33. Yang Y, Ding S, Xu G, Chen F and Ding F: MicroRNA-15a inhibition protects against hypoxia/reoxygenation-induced apoptosis of cardiomyocytes by targeting mothers against decapentaplegic homolog 7. Mol Med Rep 15: 3699-3705, 2017.

34. Xue G, Yan HL, Zhang Y, Hao LQ, Zhu XT, Mei Q and Sun SH: c-Myc-mediated repression of miR-15-16 in hypoxia is induced by increased HIF-2 $\alpha$ and promotes tumor angiogenesis and metastasis by upregulating FGF2. Oncogene 34: 1393-1406, 2015.

35. Reed JC: Regulation of apoptosis by bcl-2 family proteins and its role in cancer and chemoresistance. Curr Opin Oncol 7: 541-546, 1995. 
36. Pinkas J, Martin SS and Leder P: Bcl-2-mediated cell survival promotes metastasis of EpH4 betaMEKDD mammary epithelial cells. Mol Cancer Res 2: 551-556, 2004.

37. Planas-Silva MD, Bruggeman RD, Grenko RT and Smith JS: Overexpression of c-Myc and $\mathrm{Bcl}-2$ during progression and distant metastasis of hormone-treated breast cancer. Exp Mol Pathol 82: 85-90, 2007.

38. Ricca A, Biroccio A, Del Bufalo D, Mackay AR, Santoni A and Cippitelli M: bcl-2 over-expression enhances NF-kappaB activity and induces mmp-9 transcription in human MCF7(ADR) breastcancer cells. Int J Cancer 86: 188-196, 2000.

39. Bartel DP: MicroRNAs: Genomics, biogenesis, mechanism, and function. Cell 116: 281-297, 2004.

40. Wang Y, Li M, Zang W, Ma Y, Wang N, Li P, Wang T and Zhao G: miR-429 up-regulation induces apoptosis and suppresses invasion by targeting Bcl-2 and SP-1 in esophageal carcinoma. Cell Oncol (Dordr) 36: 385-394, 2013.
41. Wu WL, Wang WY, Yao WQ and Li GD: Suppressive effects of microRNA-16 on the proliferation, invasion and metastasis of hepatocellular carcinoma cells. Int J Mol Med 36: 1713-1719, 2015.

42. Lerner M, Harada M, Lovén J, Castro J, Davis Z, Oscier D, Henriksson M, Sangfelt O, Grandér D and Corcoran MM: DLEU2, frequently deleted in malignancy, functions as a critical host gene of the cell cycle inhibitory microRNAs miR-15a and miR-16-1. Exp Cell Res 315: 2941-2952, 2009.

(i) $\odot$ This work is licensed under a Creative Commons Attribution-NonCommercial-NoDerivatives 4.0 International (CC BY-NC-ND 4.0) License. 